\title{
Prevalência de anemia em crianças brasileiras, segundo diferentes cenários epidemiológicos ${ }^{1}$
}

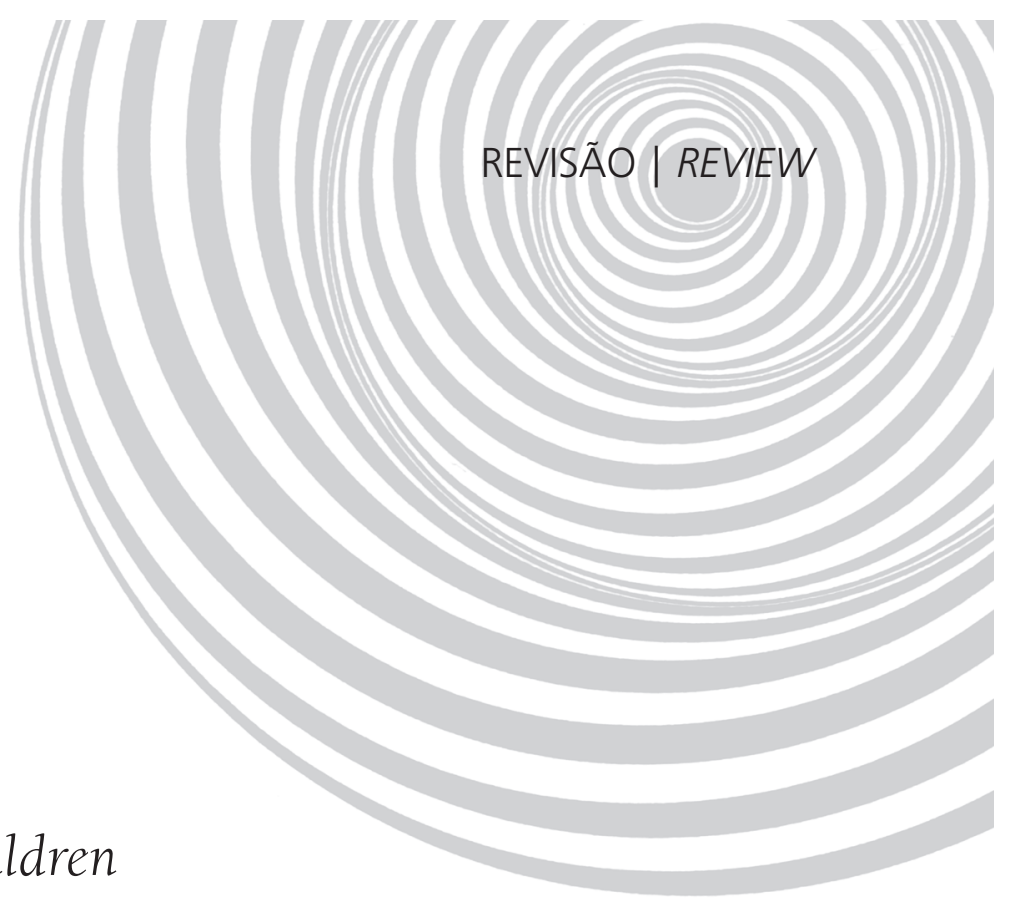

\section{Prevalence of anemia in Brazilian children in different epidemiological scenarios}

Regina Coeli da Silva VIEIRA²

Haroldo da Silva FERREIRA²

\section{R E S U M O}

Este trabalho teve por objetivo estimar a prevalência de anemia em crianças brasileiras segundo diferentes cenários epidemiológicos. Para isso, realizou-se uma revisão sistemática com metanálise dos resultados de estudos observacionais publicados nos últimos dez anos. A pesquisa de artigos foi efetuada nas bases do SciELO e PubMed, usando-se a palavra-chave "anemia" combinada com criança e Brasil. Após aplicação dos critérios de exclusão (artigos de revisão; anemia de etiologia não nutricional; diagnóstico não baseado no nível de hemoglobina $(\mathrm{Hb}<11 \mathrm{~g} / \mathrm{dL})$; amostra envolvendo crianças $>7$ anos; ausência de dados de prevalência; e não identificação do local de estudo, da amostra, da faixa etária e/ou do método diagnóstico), foram selecionados 35 artigos, posteriormente categorizados segundo a origem de suas amostras: creches/escolas $n=8$, serviços de saúde $n=12$, populações em iniquidades $n=6$ e estudos de base populacional $n=9$. Por meio de metanálise, calcularam-se a prevalência média de anemia ponderada pelos respectivos tamanhos amostrais e a razão de chances para um intervalo de confiança de $95 \%$, assumindo-se a prevalência de estudos de base populacional como referência $\mathrm{n}=1(\mathrm{CRC}=1)$, e obtiveram-se os seguintes resultados, respectivamente: creches/ escolas: $52,0 \%, 1,61$ (1,5-1,8); serviços de saúde: $60,2 \%, 2,26(2,1-2,4)$; populações em iniquidades: $66,5 \%$, $2,96(2,6-3,4)$ e estudos de base populacional: $40,1 \%$, com $p<0,0001$ para todas as comparações $\left(\chi^{2}\right)$. A anemia continua representando um grave problema de saúde pública nos distintos cenários analisados, justificando receber atenção prioritária por parte dos gestores das políticas públicas das diferentes esferas de governo no País.

Termos de indexação: Anemia. Áreas de pobreza, Brasil. Creches. Crianças. Serviços de saúde.

A B S T R A C T

This study aimed to estimate the prevalence of anemia in Brazilian children, according to different epidemiological scenarios. For this purpose, we carried out a systematic review with meta-analysis of the

\footnotetext{
1 Artigo elaborado a partir da dissertação de R.C.S. VIEIRA, intitulada "Fatores associados à prevalência de anemia em crianças menores de cinco anos do estado de Alagoas, Brasil". Universidade Federal de Alagoas; 2007.

2 Universidade Federal de Alagoas, Faculdade de Nutrição. Campus A.C. Simões, BR 104 Norte, Tabuleiro do Martins, 57072-970, Maceió, AL, Brasil. Correspondência para/Correspondence to: H.S. FERREIRA. E-mail: <haroldo.ufal@gmail.com>.
} 
434 | R.C.S. VIEIRA \& H.S. FERREIRA

results of observational studies published in the last ten years. Articles were searched in the SciELO and PubMed databases using the keyword "anemia" combined with children and Brazil. After establishing the exclusion criteria (review article, anemia not of a nutritional etiology, diagnosis not based upon the level of hemoglobin $(H b<11 \mathrm{~g} / \mathrm{dL})$, sample including children older than seven years, no prevalence data and no identification of study location, sample, age group and diagnostic method), 35 articles were selected, which were categorized according to the origin of their samples: daycare centers/schools $n=8$, health services $n=12$, populations subject to socioeconomic inequality $n=6$ and population-based studies $n=9$. A meta-analysis was used to estimate the prevalence of anemia weighted by the respective sample size, and odds ratio for a confidence interval of 95\%, assuming the prevalence of population-based studies as reference (odds ratio = 1). The following results were obtained, respectively: centers/schools: $52.0 \%, 1.61$ (1.5 to 1.8); health services: $60.2 \%, 2.26$ (2.1 to 2.4); populations subject to injustice: 66.5\%, 2.96 (2.6 to 3.4) and population-based studies: $40.1 \%$, with $p<0.0001$ for all comparisons $\left(\chi^{2}\right)$. Anemia is still a serious public health problem in the different scenarios analyzed, justifying the fact that it is still considered a priority by managers of public health policies of different government levels in the country.

Indexing terms: Anemia. Areas of poverty, Brazil. Creches. Children. Health services;

\section{N T R O D U ÇÃ O}

A anemia é definida como uma enfermidade em que o paciente apresenta um quadro de hipoxia tissular consequente de uma redução da capacidade de transporte de oxigênio pelo sangue. Essa redução na capacidade de transporte é decorrente de reduzida concentração de hemoglobina e/ou do número de eritrócitos ${ }^{1}$. Dessa forma, a anemia pode resultar de um ou mais dos seguintes fatores: (a) reduzida produção de eritrócitos; (b) elevada destruição dos eritrócitos; e (c) hemorragias e outras formas de perdas sanguíneas.

Ainda que doenças hemolíticas congênitas, tais como anemia falciforme e talassemia, sejam encontradas com maior frequência em certas populações, particularmente na África, Ásia e algumas ilhas do Oceano Pacífico, e que infestações parasitárias e infecções desempenhem função importante na etiologia da anemia em países tropicais, as deficiências nutricionais são as principais causas de anemia no mundo².

A anemia nutricional é um estado no qual a concentração de Hemoglobina $(\mathrm{Hb})$ no sangue está abaixo dos níveis considerados normais para idade, gênero, estado fisiológico e altitude como consequência de uma deficiência de nutrientes essenciais, independentemente da causa dessa deficiência ${ }^{3}$. Do ponto de vista epidemiológico, a causa mais frequente decorre da deficiência de ferro, caracterizando a chamada anemia ferropriva ou ferropênica. Estima-se que 50\% a 90\% de to- dos os tipos de anemia no mundo ocorram pela deficiência de ferro 4 .

A prevalência de anemia divulgada para o Brasil a partir de dados da Pesquisa Nacional de Demografia e Saúde da Criança e da Mulher (PNDS) $^{5}$ foi de $20,9 \%$. Apesar da importância inquestionável desse estudo, o primeiro de abrangência nacional, tal resultado não discrimina situações específicas em que prevaleçam diferenciais importantes, conforme os distintos contextos epidemiológicos. Estudos recentes e pontuais têm encontrado valores superiores ao observado pela PNDS-2006, indicando, ainda, tendência de aumento da prevalência em crianças ${ }^{6,7}$, de modo que a anemia é considerada hoje a carência nutricional mais prevalente no Brasil, superando a desnutrição energético-proteica ${ }^{7,8}$.

A anemia na infância pode provocar dificuldades na aprendizagem da linguagem, distúrbios psicológicos e comportamentais, além de debilitar a defesa imunológica, facultando a ocorrência e/ou agravamento de doenças infecciosas ${ }^{9}$.

Em virtude da importância da anemia em crianças como problema de saúde pública, este trabalho teve por objetivo propiciar estimativas para sua prevalência, conforme diferentes cenários epidemiológicos.

\section{M É T O D O S}

Para a consecução do objetivo proposto, procedeu-se a uma revisão sistemática da lite- 
ratura, seguida de metanálise, de acordo com o método Moose ${ }^{10}$. A Figura 1 esquematiza a sequência de fases até a elaboração da análise estatística.

A identificação dos artigos de interesse foi realizada utilizando-se as bases do SciELO (http:// www.scielo.org/php/index.php) e do do PubMed da U.S. National Library of Medicine (http:// www.ncbi.nlm.nih.gov/pubmed/), a partir da palavra-chave pré-definida "anemia" combinada com "criança" ou "children" e "Brasil" ou "Brazil", limitando a busca para palavras do título ou do resumo, nos idiomas português ou inglês, estudos com seres humanos e publicações a partir de 1996. Para garantir a qualidade dos artigos, só foram incluídas publicações indexadas nessas bases de dados.

Numa segunda etapa, foram aplicados os critérios de exclusão, considerando inadequados para os objetivos propostos os artigos de revisão de literatura, os que abordaram anemia de etiologia definidamente não nutricional, os que adotaram critério diagnóstico não baseado na quantificação da hemoglobina, aqueles cuja amostra não era referente a crianças com idade inferior a sete anos, os que não dispunham de dados de prevalência, além daqueles que não apresentavam identificação precisa do local de estudo, da amostra, da faixa etária e do método de diagnóstico.

Autor 1 e 2: Planejamento metodológico: definição das bases de dados (PubMed e SciELO), das palavras-chave (anemia, criança e Brasil), período a ser pesquisado (últimos 10 anos) e critérios de inclusão e exclusão.

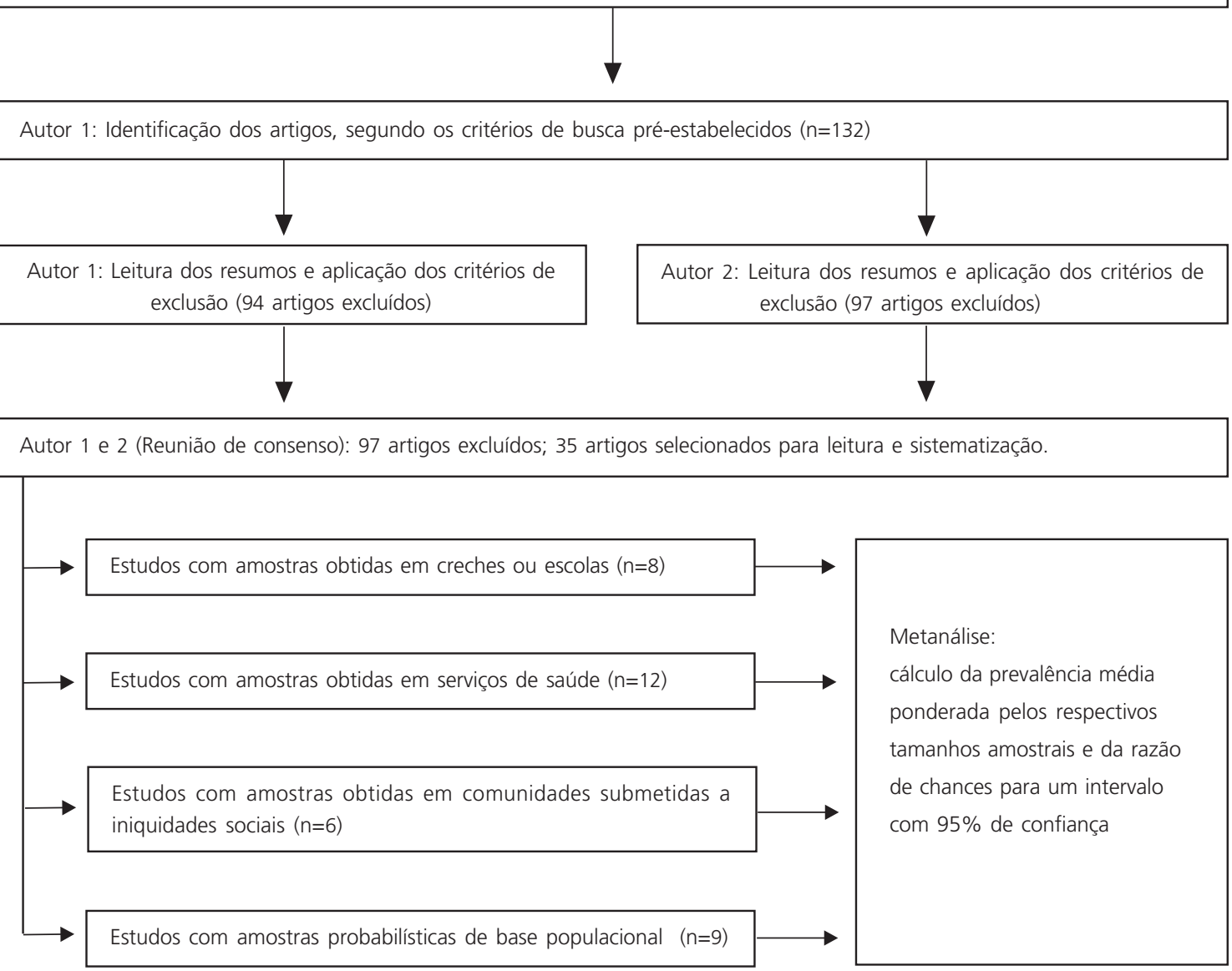

Figura 1. Fluxograma utilizado para sistematização e metanálise de resultados de estudos sobre prevalência de anemia em crianças realizados no Brasil nos últimos dez anos. 
436 | R.C.S. VIEIRA \& H.S. FERREIRA

Resumos de artigos cujo conteúdo possibilitasse sua exclusão pelos critérios pré-definidos não tinham o texto completo selecionado para passar à fase seguinte. Já os que ultrapassaram esse processo foram submetidos à leitura e à sistematização dos resultados e, em seguida, agrupados em quatro categorias conforme a natureza de suas respectivas amostras: escolas/creches, serviços de saúde, populações em iniquidades sociais (comunidades indígenas, assentamentos rurais, favelas, clientela da Pastoral da Criança) e, por fim, estudos de base populacional representativos de cidades, regiões ou estados brasileiros.

Foram calculados para cada uma dessas quatro categorias a prevalência média ponderada pelos respectivos tamanhos amostrais, a amplitude e a Razão de Chances (RC) para um intervalo de confiança de 95\% (IC95\%), assumindo como prevalência de referência aquela encontrada para os estudos de base populacional $(R C=1)$. A significância das diferenças $(p<0,05)$ entre as frequências encontradas foi verificada por meio do teste qui-quadrado.

\section{RESULTADOS E DISCUSSÃO}

Foram identificados 132 artigos. Após a aplicação dos critérios de exclusão, 35 trabalhos foram considerados adequados e tiveram seus resultados sistematizados. Destes, nove foram classificados como de base populacional, oito envolveram amostras obtidas em creches ou escolas, seis foram realizados com populações em situação de iniquidades e doze foram procedentes de serviços de saúde.

\section{Prevalência de anemia em amostras obtidas em escolas ou creches}

Foram incluídos oito artigos, envolvendo amostras que variaram de 135 a 865 crianças. A menor prevalência encontrada foi de $35,0 \%$ e a maior de $68,8 \%$. A prevalência média ponderada pelo tamanho amostral foi de $52,0 \%$. A Tabela 1 sumariza os resultados encontrados.

De modo geral, a partir dos seis meses, quanto menor a idade, maior o risco de anemia, de forma que essa variável tem sido referida como o principal fator biológico associado a esse agravo, possivelmente, devido a diferenciais na velocidade de crescimento, aumentando a demanda nutricional e, consequentemente, uma maior susceptibilidade ao desenvolvimento da anemia quando em circunstâncias de ingestão deficiente de ferro. Dietas excessivamente baseadas em consumo de leite de vaca podem ser uma das causas do alto risco de anemia nos primeiros anos de vida, por esse ser um alimento pobre em ferro ${ }^{11}$.

Tabela 1. Prevalência de anemia em crianças, segundo estudos publicados a partir de 1996, envolvendo amostras obtidas em escolas ou creches situadas no Brasil.

\begin{tabular}{|c|c|c|c|c|c|}
\hline Fonte & Local de estudo & $\begin{array}{c}\text { Faixa etária } \\
\text { (meses) }\end{array}$ & $\begin{array}{l}\text { Tamanho } \\
\text { amostral }\end{array}$ & $\begin{array}{c}\text { Recurso } \\
\text { diagnóstico }\end{array}$ & $\begin{array}{c}\text { Prevalência } \\
(\%)\end{array}$ \\
\hline Brunken et al. ${ }^{8}$ & Creches públicas de Cuiabá (MT) & $<36$ & 271 & Hemograma & 63,1 \\
\hline Konstantyner et al. ${ }^{12}$ & Creches públicas ou filantrópicas de São Paulo (SP) & $<24$ & 212 & HemoCue & 51,9 \\
\hline Spinelli et al. ${ }^{13}$ & Uma creche pública de São Paulo (SP) & 3 a 72 & 135 & HemoCue & 35,0 \\
\hline Brunken et al..$^{14}$ & Seis creches públicas de Cuiabá (MT) & $<36$ & 178 & HemoCue & 41,0 \\
\hline Silva et al. ${ }^{15}$ & Todas as escolas municipais infantis de Porto Alegre (RS) & 0 a 36 & 557 & HemoCue & 47,8 \\
\hline Almeida et al. ${ }^{16}$ & Creches municipais de Pontal (SP) & 12 a 72 & 192 & Hemograma & 62,5 \\
\hline Bueno et al. ${ }^{17}$ & 20 creches públicas de São Paulo (SP) & 6 a 75 & 330 & HemoCue & 68,8 \\
\hline Matta et al. ${ }^{18}$ & Creches públicas do Rio de Janeiro (RJ) & $<60$ & 865 & HemoCue & 47,3 \\
\hline \multicolumn{3}{|c|}{ Média ponderada pelo tamanho amostral } & 2740 & & 52,0 \\
\hline
\end{tabular}


Além da idade, outros fatores estão envolvidos na etiologia do problema, pois diferentes estudos abrangendo crianças da mesma faixa etária matriculadas em creches têm encontrado diferentes prevalências.

Konstantyner et al. ${ }^{12}$, avaliando crianças menores de dois anos de creches públicas ou filantrópicas de São Paulo (SP), encontraram quase $20,0 \%$ mais anemia que Spinelli et al..$^{13} \mathrm{em}$ uma creche da mesma cidade. Brunken et al. ${ }^{8}$, com dados de crianças menores de 36 meses que frequentavam creches públicas de Cuiabá, observaram que $63,1 \%$ delas tinham anemia. Revelaram que embora fontes de ferro (carnes e feijões) fizessem parte do consumo alimentar diário, provavelmente não ocorriam na proporção devida, além de serem ingeridos concomitantemente com fatores antinutricionais presentes nos chás que eram rotineiramente oferecidos, além do xarope à base de guaraná, muito comum na região. Estudo posterior, também em creches no mesmo município, encontrou $41 \%$ de anemia, representando uma redução de cerca de um terço em relação ao estudo anterior. Certamente o "alerta" sinalizado pelos dados então revelados proporcionou maior atenção ao problema, resultando numa melhoria razoável da situação ${ }^{14}$.

Silva et al..$^{15}$ estudaram crianças das escolas municipais de Porto Alegre (RS) aleatoriamente selecionadas e relataram que o baixo nível socioeconômico (renda per capita igual ou inferior a um salário-mínimo), a faixa etária de 12 a 23 meses e a presença de dois ou mais irmãos com menos de cinco anos foram os principais fatores determinantes da condição de anemia.

Almeida et al. ${ }^{16}$, ao estudar fatores determinantes de anemia e deficiência de ferro em crianças de duas creches da cidade de Pontal (SP), encontraram $62,5 \%$ de anemia, prevalência semelhante à verificada por Bueno et al. ${ }^{17}$ creches públicas do município de São Paulo (68,8\%). Em ambas as pesquisas, a anemia foi superior em mais de $15,0 \%$ ao valor encontrado em creches da capital do Rio de Janeiro ${ }^{18}$.

\section{Prevalência de anemia em amostras obtidas em serviços de saúde}

Foram incluídos 12 artigos, envolvendo amostras que variaram de 110 a 5146 crianças. A menor prevalência encontrada foi de 55,1\% e a maior de $89,1 \%$. A prevalência média ponderada pelo tamanho amostral foi de $60,2 \%$ (Tabela 2).

É plausível supor que estudos que envolvam amostras obtidas em serviços de saúde tendam a revelar maiores prevalências de anemia em relação à população geral em virtude de abranger, com maior probabilidade, pessoas debilitadas e portadoras de agravos que predisponham a essa carência nutricional.

Analisando crianças entre seis e doze meses em uma unidade pública de saúde de Goiânia, Hadler et al..$^{19}$ encontraram 60,9\% com anemia. Silva et al. ${ }^{20}$, Silva et al..$^{21}$ e Miranda et al. ${ }^{22}$ encontraram valores semelhantes em serviços de saúde da rede pública de Viçosa (MG).

No município de Angatuba (SP), das 269 crianças de 6 a 42 meses matriculadas no Programa de Suplementação Alimentar da Secretaria de Estado da Saúde e da Prefeitura Municipal, $62,3 \%$ apresentaram anemia. Porém, após seis meses, esse percentual reduziu-se para $41,8 \%$ e, ao final de um ano, para $26,4 \%{ }^{23}$. Em instituições de cuidado diário na cidade de Pontal (SP), eram anêmicas $68,7 \%$ das 115 crianças de 12 a 72 meses avaliadas 24 .

No sul do País, no único hospital da cidade de São Leopoldo (RS), mais de $60 \%$ dos recém-nascidos tinham anemia ${ }^{25}$. A situação parece não mudar muito em unidades de saúde do município de Maringá (PR) ${ }^{26}$. É importante considerar, todavia, o maior risco de anemia existente nessa faixa etária específica.

Em estudo realizado no outro extremo do País ${ }^{27}$, avaliaram-se crianças de 6 a 24 meses atendidas em um centro de cuidado primário de Belém (PA), verificando-se que 55,1\% eram anê- 
438 | R.C.S. VIEIRA \& H.S. FERREIRA

Tabela 2. Prevalência de anemia em crianças, segundo diversos estudos publicados a partir de 1996, envolvendo amostras obtidas em serviços de saúde situados no Brasil.

\begin{tabular}{|c|c|c|c|c|c|}
\hline Fonte & Local de estudo & $\begin{array}{l}\text { Faixa etária } \\
\text { (meses) }\end{array}$ & $\begin{array}{l}\text { Tamanho } \\
\text { amostral }\end{array}$ & $\begin{array}{c}\text { Recurso } \\
\text { diagnóstico }\end{array}$ & $\begin{array}{l}\text { Prevalência } \\
(\%)\end{array}$ \\
\hline Hadler et al. ${ }^{19}$ & Unidade pública de saúde de Goiânia (GO) & 6 a 12 & 110 & Hemograma & 60,9 \\
\hline Silva et al. ${ }^{20}$ & Rede pública de saúde do município de Viçosa (MG) & 6 a 12 & 204 & HemoCue & 60,8 \\
\hline Silva et al. ${ }^{21}$ & Serviço de saúde da rede pública de Viçosa (MG) & 6 a 12 & 205 & HemoCue & 57,6 \\
\hline Miranda et al. ${ }^{22}$ & Serviço de saúde da rede pública de Viçosa (MG) & 12 a 60 & 171 & HemoCue & 63,2 \\
\hline Torres et al. ${ }^{23}$ & Programa de Suplementação Alimentar, Angatuba (SP) & 6 a 42 & 269 & HemoCue & 62,3 \\
\hline $\begin{array}{l}\text { Nogueira-de-Almeida } \\
\text { et al. }{ }^{24}\end{array}$ & Instituições de cuidado diário na cidade de Pontal (SP) & 12 a 72 & 115 & Hemograma & 68,7 \\
\hline Vitolo et al. ${ }^{25}$ & Hospital Centenário, São Leopoldo (RS) & 0 a 1 & 500 & Hemograma & 60,0 \\
\hline Uchimura et al. ${ }^{26}$ & Unidades de saúde do Município de Maringá (PR) & $<12$ & 587 & HemoCue & 58,0 \\
\hline Neves et al. ${ }^{27}$ & Centro de cuidado primário em Belém (PA) & 6 a 24 & 365 & $\begin{array}{l}\text { Cianometa- } \\
\text { hemoglobina }\end{array}$ & 55,1 \\
\hline Leal \& Osório ${ }^{28}$ & Instituto Materno Infantil de Pernambuco, Recife (PE) & 6 a 23 & 402 & Hemograma & 89,1 \\
\hline Spinelli et al. ${ }^{29}$ & Clínicas públicas de 12 municípios das 5 regiões do Brasil & 6 a 12 & 2715 & HemoCue & 65,4 \\
\hline Szarfarc et al. ${ }^{30}$ & $\begin{array}{l}\text { Serviços públicos de saúde de cidades das cinco regiões } \\
\text { geográficas brasileiras }\end{array}$ & $<12$ & 5146 & HemoCue & 55,6 \\
\hline \multicolumn{3}{|c|}{ Média ponderada pelo tamanho amostral } & 10789 & & 60,2 \\
\hline
\end{tabular}

micas. Na região Nordeste, Leal \& Osório ${ }^{28}$ encontraram prevalência de $89,1 \%$ em crianças de 6 a 23 meses que frequentavam o Instituto Materno Infantil de Pernambuco.

Spinelli et al. ${ }^{29}$, num estudo transversal com residentes de 12 municípios das cinco regiões do Brasil envolvendo crianças atendidas em clínicas públicas, verificaram que das 2715 crianças de 6 a 12 meses investigadas, 65,4\% apresentavam anemia. Estudo posterior com ampliação da amostra para 5146 crianças menores de 12 meses, desenvolvido pelo mesmo grupo ${ }^{30}$, encontrou uma prevalência de 55,6\%.

\section{Prevalência de anemia em amostras obtidas de populações em iniquidades sociais}

Foram incluídos sete artigos envolvendo amostras que variaram de 69 a 369 crianças. A menor prevalência encontrada foi de $47,5 \%$ e a maior de $96,4 \%$. A prevalência média pondera- da pelo tamanho amostral foi de 63,5\% (Tabela 3).

Em assentamento rural no Vale do Rio Doce (MG), estudo com crianças menores de 60 meses detectou $47,5 \%$ de anemia ${ }^{31}$. Morais et al. ${ }^{32}$, no Mato Grosso do Sul, encontraram uma prevalência de anemia em crianças indígenas superior a $85,0 \%$ entre aquelas de 6 a 24 meses e $50,8 \%$ entre as de 24 e 60 meses.

Outros estudos com menores de cinco anos nessas comunidades de alto risco encontraram prevalências exorbitantes, tal como as verificadas nas aldeias Suruí da Amazônia $(84,0 \%)^{33}$ e em crianças de uma favela da periferia de Maceió (AL) $(96,0 \%)^{34}$.

No Rio Grande do Sul, tanto em estudo realizado em comunidades atendidas pela Pastoral da Criança em Pelotas ${ }^{35}$ quanto em famílias de baixo nível socioeconômico do município de São Leopoldo, a prevalência de anemia foi maior que $50,0 \%$; nesta última cidade, $18,3 \%$ apresentaram a forma grave ${ }^{36}$. 
Prevalência de anemia em estudos de base populacional representativos de cidades, regiões ou estado

Foram incluídos nove artigos com amostras que variaram de 254 a 1280 crianças. A menor prevalência encontrada foi de $22,2 \%$ e a maior de $54,0 \%$. A prevalência média ponderada pelo tamanho amostral foi de 40,1\% (Tabela 4).

Assis et al. ${ }^{37}$ avaliaram crianças de áreas urbanas de municípios da região do semi-árido baiano e detectaram $22,2 \%$ de anemia, enquanto na capital do Estado quase $50 \%$ das crianças apresentavam a doença ${ }^{38}$, valor superior ao observado em crianças pernambucanas, que foi de $40,9 \%{ }^{39}$.

Na área urbana de dois municípios da Amazônia Ocidental Brasileira, Muniz et al. ${ }^{40}$ encontraram anemia em cerca de $30 \%$ das crianças em idade pré-escolar.

Estudando crianças na cidade de São Paulo (SP), Monteiro et al. ${ }^{6}$ relatam um adicional de 11,3\% na prevalência de anemia no intervalo entre 1984/1985 e 1995/1996. As prevalências encon-

Tabela 3. Prevalência de anemia em crianças, segundo diversos estudos publicados nos últimos 10 anos, envolvendo amostras obtidas em populações em iniquidades sociais situadas no Brasil.

\begin{tabular}{|c|c|c|c|c|c|}
\hline Fonte & Local de estudo & $\begin{array}{l}\text { Faixa etária } \\
\text { (meses) }\end{array}$ & $\begin{array}{l}\text { Tamanho } \\
\text { amostral }\end{array}$ & $\begin{array}{c}\text { Recurso } \\
\text { diagnóstico }\end{array}$ & $\begin{array}{l}\text { Prevalência } \\
\text { (\%) }\end{array}$ \\
\hline Castro et al. ${ }^{31}$ & Assentamento rural no Vale do Rio Doce (MG) & 0 a 60 & 69 & $\begin{array}{l}\text { Hemoglobinômetro } \\
\text { portátil }\end{array}$ & 47,5 \\
\hline Morais et al. ${ }^{32}$ & $\begin{array}{l}\text { Aldeias Terenas de Limão Verde e Córrego Seco, Aqui- } \\
\text { dauana (MS) }\end{array}$ & 6 a 60 & 108 & HemoCue & 64,8 \\
\hline Orellana et al. ${ }^{33}$ & Reserva indígena (RO e MG) & 6 a 59 & 144 & HemoCue & 84,0 \\
\hline Ferreira et al. ${ }^{34}$ & Favela "Cidade de Lona", Maceió (AL) & 6 a 60 & 137 & $\begin{array}{l}\text { Haemoglobin } \\
\text { colour scale }\end{array}$ & 96,4 \\
\hline Santos et al. ${ }^{35}$ & $\begin{array}{l}\text { Clientela da Pastoral da Criança na periferia de Pelotas } \\
\text { (RS) }\end{array}$ & $<72$ & 304 & HemoCue & 53,0 \\
\hline Vitolo \& Bortolini ${ }^{36}$ & $\begin{array}{l}\text { Famílias de baixo nível socioeconômico, São Leopoldo } \\
\text { (RS) }\end{array}$ & 6 e 12 & 369 & Hemograma & 63,7 \\
\hline \multicolumn{2}{|c|}{ Média ponderada pelo tamanho amostral } & & 1131 & & 66,5 \\
\hline
\end{tabular}

Tabela 4. Prevalência de anemia em crianças, segundo diversos estudos publicados nos últimos 10 anos, envolvendo amostras representativas de cidades, regiões ou estados no Brasil.

\begin{tabular}{|c|c|c|c|c|c|}
\hline Fonte & Local de estudo & $\begin{array}{c}\text { Faixa etária } \\
\text { (meses) }\end{array}$ & $\begin{array}{l}\text { Tamanho } \\
\text { amostral }\end{array}$ & $\begin{array}{c}\text { Recurso } \\
\text { diagnóstico }\end{array}$ & $\begin{array}{c}\text { Prevalência } \\
(\%)\end{array}$ \\
\hline Monteiro et al. ${ }^{6}$ & São Paulo (SP) & $<60$ & 1280 & HemoCue & 46,9 \\
\hline $\begin{array}{l}\text { Levy-Costa \& } \\
\text { Monteiro }{ }^{11}\end{array}$ & São Paulo (SP) & $<60$ & 854 & HemoCue & 45,2 \\
\hline Assis et al. ${ }^{37}$ & Áreas urbanas de sete municípios da região semi-árida (BA) & 1 a 74 & 745 & $\begin{array}{l}\text { Cianometa- } \\
\text { hemoglobina }\end{array}$ & 22,2 \\
\hline Assis et al. ${ }^{38}$ & Salvador (BA) & 6 a 72 & 603 & HemoCue & 46,3 \\
\hline Osório et al. ${ }^{39}$ & Pernambuco & 6 a 59 & 777 & HemoCue & 40,9 \\
\hline Muniz et al. ${ }^{40}$ & Área urbana de Assis Brasil e Acrelândia (AC) & $<60$ & 677 & HemoCue & 30,6 \\
\hline Duarte et al. ${ }^{41}$ & Área urbana de Itupeva (SP) & $<24$ & 254 & HemoCue & 41,7 \\
\hline Neumann et al. ${ }^{42}$ & Área urbana do Município de Criciúma (SC) & $<36$ & 476 & fotômetro BMS & 54,0 \\
\hline Assunção et al. ${ }^{43}$ & Pelotas (RS) & $<60$ & 453 & HemoCue & 30,2 \\
\hline Média ponderada & pelo tamanho amostral & & 6119 & & 40,1 \\
\hline
\end{tabular}


tradas em trabalhos subsequentes no mesmo Estado ${ }^{11,41}$ demonstram que a situação pouco se alterou, ficando em torno dos $40 \%$.

Estudos realizados em Criciúma (SC) ${ }^{42}$ e em Pelotas (RS) ${ }^{43}$, no Sul do País, encontraram prevalências distintas embora elevadas (54\% vs Pelotas $30,2 \%)$.

A Tabela 5 consolida os resultados encontrados para as quatro categorias de investigações avaliadas. Assumindo-se a prevalência observada a partir dos estudos de base populacional como referência $(40,1 \%)$, verificou-se que os piores resultados foram encontrados entre crianças pertencentes às populações em situação de iniquidade social (66,5\%), sendo um resultado surpreendente, pois, como foi anteriormente argumentado, seria esperada uma maior prevalência entre as crianças alocadas nos serviços de saúde $(60,2 \%)$, pois elas apresentariam maiores risco de estarem doentes e susceptíveis à anemia. A chance de uma criança ser anêmica por pertencer a tais populações foi cerca de 30,0\% maior em relação às crianças atendidas nos serviços de saúde e quase o triplo em relação à população geral.

A prevalência verificada nas escolas e creches também foi significativamente superior à encontrada na população em geral. O fato de a maior parte desses estudos envolver crianças de creche - e, portanto, um maior contingente de indivíduos na faixa etária de maior risco $(<2$ anos) - pode explicar parte desse resultado. No entanto, estando essas crianças ao abrigo do poder público ou de um serviço especializado, infere-se que essas instituiç̧ões não estão atuando de forma adequada para prevenir e controlar esse agravo em sua clientela. Todavia, estudos específicos são necessários para melhor fundamentar essa interpretação, pois a análise aqui realizada não teve como considerar a condição em que a criança chegou à creche nem se o tempo de frequência era suficiente para reverter um quadro de anemia pré-existente.

É importante ressaltar que a alta prevalência encontrada nos estudos de base populacional $(40,1 \%)$ reflete a manutenção das condições que determinam que a anemia permaneça sendo um problema de alta relevância na população brasileira. Adicionalmente, de acordo com Muniz et al. ${ }^{40}$, avaliações que consideram a medida isolada de hemoglobina como critério diagnóstico subestimam a magnitude da deficiência de ferro, visto que em sua pesquisa apenas $47,6 \%$ das crianças com deficiência de ferro tinham anemia.

Há décadas diversos estudos vêm apontando a anemia como um dos problemas nutricionais de maior magnitude no mundo ${ }^{5,9}$. Em 1985, a Organização Mundial da Saúde (OMS) estimou em 30,0\% a prevalência de anemia na população mundial; evidenciando, porém, diferenciais importantes segundo os distintos contextos geopolíticos e de faixas etárias. Seriam anêmicas $43,0 \%$ das crianças de zero a quatro anos de idade, sendo $12,0 \%$ entre as que habitavam países desenvolvidos e $51,0 \%$ entre as que viviam em países em desenvolvimento ${ }^{44}$.

Nossos dados revelaram prevalências que variaram de $22,2 \%$ a $96,4 \%$, semelhante ao observado no Caribe de língua inglesa, onde a prevalência de anemia modificava-se muito de um lugar para outro e flutuava de 15,0\% a 80,0\% ${ }^{45}$.

Tabela 5. Síntese dos resultados obtidos em estudos sobre prevalência de anemia realizados com crianças no Brasil a partir de 1996, categorizados segundo procedência das amostras.

\begin{tabular}{lccccc}
\hline Proveniência da amostra & $\begin{array}{c}\text { Número de } \\
\text { estudos }\end{array}$ & $\begin{array}{c}\text { Amostra } \\
(\mathrm{n})\end{array}$ & $\begin{array}{c}\text { Prevalência (\%) } \\
\text { (Amplitude) }\end{array}$ & $\begin{array}{c}\text { Razão de chances } \\
(\text { IC95\%) }\end{array}$ & $\begin{array}{c}\text { Valor } p \\
\left(\chi^{2}\right)\end{array}$ \\
\hline Base populacional & 9 & 6119 & $40,1(54,0-22,2=31,8)$ & 1 & - \\
Escolas ou creches & 8 & 2740 & $52,0(68,8-35,0=33,8)$ & $1,61(1,47-1,77)$ & $<0,0001^{*}$ \\
Populações em iniquidades & 6 & 1131 & $66,5(96,4-47,5=48,9)$ & $2,96(2,58-3,39)$ & $<0,0001^{*}$ \\
Serviços de saúde & 12 & 10789 & $60,2(89,1-55,1=34,0)$ & $2,26(2,11-2,41)$ & $<0,0001^{*}$ \\
\hline
\end{tabular}

IC95\%: Intervalo de confiança a 95\%; 'Diferença estatisticamente significativa em relação à prevalência estimada a partir dos estudos de base populacional. 
Quando a prevalência da anemia é superior a $40 \%$, a causa mais comum é a deficiência de ferro dietético devido à baixa ingestão de fontes desse micronutriente e/ou ao alto consumo de inibidores de sua absorção ${ }^{3}$, fatores que devem ser considerados no contexto das ações de prevenção e controle.

\section{CONSIDERAÇÕ ES FIN A IS}

Com base nos dados aqui analisados, pode-se estimar que a prevalência de anemia em crianças brasileiras atinge níveis superiores a 40,0\%, tratando-se, portanto, de um problema de grave significância populacional ${ }^{46} \mathrm{e}$, em termos de magnitude, o principal problema carencial do País ${ }^{47}$. A consistência de tal achado é evidenciada a partir de estudo recentemente conduzido com amostra probabilística representativa dos pré-escolares do estado de Alagoas, no qual se observou prevalência de $45,0 \%{ }^{48}$. É importante destacar, todavia, que, em determinados contextos, a situação é ainda mais preocupante, conforme demonstrado para as populações em evidente condição de iniquidade social.

Quanto à discrepância em relação à prevalência encontrada pela PNDS de $2006^{5}(20,9 \%)$, torna-se necessário aguardar por uma publicação específica desse estudo para melhor embasar a discussão. Algumas questões, todavia, podem ser destacadas. A relativamente baixa prevalência encontrada seria justificada pela consequência das ações do Programa Nacional de Suplementação de Ferro, com ações como a suplementação semanal de ferro ${ }^{49} \mathrm{e}$ a fortificação das farinhas de trigo e milho com ferro e ácido fólico ${ }^{50}$. Embora essa seja uma explicação possível, parece pouco plausível, pois o mesmo resultado não tem sido encontrado por outros estudos de base populacional conduzidos à mesma época11,38,40,41,43,48. Assim, apesar das importantes medidas adotadas, os dados ora apresentados parecem indicar que mais esforços serão necessários para que a anemia desapareça como problema de saúde pública em nossa população.
Um outro aspecto a ser considerado diz respeito às diferenças metodológicas. Estudos epidemiológicos têm utilizado, em sua grande maioria, o HemoCue para quantificação do nível de hemoglobina, embora outros recursos venham sendo também utilizados. Na PNDS-2006 ${ }^{5}$, os níveis de hemoglobina foram aferidos a partir de uma amostra de sangue, coletada com microlanceta, depositada em papel-filtro e analisada pelo método da cianometa-hemoglobina (http:// bvsms.saude.gov.br/bvs/pnds/anemia.php). Embora não seja objetivo deste estudo discutir a validade dos diferentes recursos para diagnóstico, diferenças metodológicas podem explicar resultados conflitantes.

Uma explicação adicional para justificar essa aparente discrepância nas prevalências divulgadas seria aquela que motivou a própria elaboração deste artigo. Como a PNDS-2006 trabalhou com amostra representativa de crianças de todo o território nacional, é possível que populações de estados não contempladas nos estudos aqui sistematizados e onde a prevalência de anemia seja inferior a 20,0\% tenham contribuído para "puxar" para baixo a média geral encontrada. Provavelmente, o planejamento amostral da PNDS-2006 não permita discriminar os resultados por estados. No entanto, essa informação poderia esclarecer melhor essa possibilidade. De qualquer forma, isso enfatiza a importância de se considerar os distintos contextos em que serão realizadas as ações de prevenção e controle, trabalhando-se com números mais próximos da realidade das populações-alvo, permitindo melhor planejamento e avaliação das ações.

Ao comprometer o fornecimento do oxigênio para os tecidos, a anemia afeta o metabolismo energético, o crescimento, diferenciação celular, mielogênese, competência imunológica, absorção e biotransformação. Provoca retardo mental, reduzido desempenho cardíaco e ineficiência na capacidade de realizar trabalho ${ }^{51}$. A anemia induz, portanto, prejuízo no rendimento escolar, acuidade mental e capacidade de concentração. As consequências para o sistema nervoso são das mais preocupantes ${ }^{52}$. Tais aspectos eviden- 
442 | R.C.S. VIEIRA \& H.S. FERREIRA

ciam os danos causados pela anemia à saúde e desenvolvimento da criança, revelando a urgência no controle desse agravo na população.

A anemia ferropriva pode ser prevenida pela ingestão habitual de alimentos fontes de ferro, principalmente sob a forma heme. Ao consumir alimentos fontes de ferro não heme, deve-se associá-los à ingestão concomitante de fontes de ácido ascórbico ${ }^{53}$ a fim de melhorar sua biodisponibilidade por reduzir o efeito inibidor provocado por polifenóis e fitatos ${ }^{54}$. Conhecer e monitorar os hábitos alimentares da população e desenvolver ações educativas objetivando garantir a adoção de uma alimentação adequada não só são medidas importantes na prevenção da anemia, mas também de uma infinidade de outros agravos à saúde. Todavia tais ações só poderão ser efetivadas a partir da inclusão do profissional nutricionista nas equipes de atenção básica de saúde, o que, no Brasil, vem sendo procedido de forma ainda bastante modesta, contrariando as propostas aprovadas na III Conferência Nacional de Segurança Alimentar e Nutricional, ocorrida em julho de 2007 em Fortaleza (CE), com respaldo direto de 1333 delegados da sociedade civil e de governos (federal, estadual e municipal) e, indiretamente, por mais de 70 mil pessoas envolvidas na realização de conferências preparatórias estaduais, sub-regionais e municipais nos 26 Estados e no Distrito Federal ${ }^{55}$.

\section{CONCLUSÃ O}

Conclui-se que, no Brasil, a anemia continua a representar um grave problema de saúde pública que afeta milhões de crianças, causando sérios danos ao seu crescimento, desenvolvimento e saúde geral. Assim, os gestores de políticas públicas deveriam reconhecer o controle dessa situação como uma questão de alta prioridade dos municípios, dos estados e do país. Uma alternativa seria a adoção das propostas aprovadas na III Conferência Nacional de Segurança Alimentar e Nutricional, o que, seguramente, iria elevar a eficácia das ações que já vêm sendo empreendidas.

\section{COLABORADORES}

R.C.S. VIEIRA com todas as etapas do trabalho e redigiu a versão inicial do artigo. H.S. FERREIRA contribuiu com a revisão final do manuscrito e da orientação do trabalho desde a sua concepção.

\section{REFER Ê N CIAS}

1. Shah A. Anemia. Indian J Med Sci. 2004; 58:24-5.

2. De Maeyer EM, Dallman P, Gurney JM, Hallberg L, Sood SK, Srikantia SG. Preventing and controlling iron deficiency anaemia through primary health care: a guide for health administrators and programme managers. Geneva: WHO; 1989.

3. World Health Organization. Nutritional anemia: report of a WHO scientific group. Geneva: WHO; 1968. Technical Report Series, 405.

4. Stoltzfus RJ. Iron deficiency: global prevalence and consequences. Food Nutr Bull. 2003; 24(4 Suppl): S99-103.

5. Brasil. Ministério da Saúde. PNDS 2006. Relatório da pesquisa nacional de demografia e saúde da criança e da mulher. Brasília; 2008 [acesso 2008 ago 7]. Disponível em: <http://www.saude.gov.br/ pnds2006>.

6. Monteiro CA, Szarfarc SC, Mondini L. Tendência secular da anemia na infância na cidade de São Paulo (1984-1996). Rev Saúde Pública. 2000; 34(6): 62-72. doi: 10.1590/S0034-891020000007000 09.

7. Batista Filho MO. controle das anemias no Brasil. Rev Bras Saúde Mater Infant. 2004; 4(2):121-3. doi: 10.1590/S1519-38292004000200001.

8. Brunken GS, Guimarães LV, Fisberg M. Anemia em crianças menores de 3 anos que frequentam creches públicas em período integral. J Pediatr. 2002; 78(1):50-6. doi: 10.1590/S0021-75572002000 100011.

9. Olivares M, Walter T. Causas y consecuencias de la deficiencia de hierro. Rev Nutr. 2004; 17(1):5-14. doi: 10.1590/S1415-52732004000100001.

10. Stroup DF, Berlin JA, Morton SC, Olkin I, Williamson $G D$, Rennie D, et al. Meta-analysis of Observational Studies in Epidemiology. JAMA. 2000; 283(15): 2008-12.

11. Levy-Costa RB, Monteiro CA. Consumo de leite de vaca e anemia na infância no Município de São Paulo. Rev Saúde Pública. 2004; 38(6):797-803. doi: 10.1590/S0034-89102004000600007.

12. Konstantyner T, Taddei JAAC, Palma D. Fatores de risco de anemia em lactentes matriculados em 
creches públicas ou filantrópicas de São Paulo. Rev Nutr. 2007; 20(4):349-59. doi: 10.1590/\$1415-52 732007000400002.

13. Spinelli MGN, Souza JMP, Souza SB, Sesoko EH. Confiabilidade e validade da palidez palmar e de conjuntivas como triagem de anemia. Rev Saúde Pública. 2003; 37(4):404-8. doi: 10.1590/S0034-89 102003000400003.

14. Brunken GS, Muniz PT, Silva SM. Weekly iron supplementation reduces anemia prevalence by 1/3 in preschool children. Rev Bras Epidemiol. 2004; 7(2):210-9. doi: 10.1590/S1415-790X2004 000200010.

15. Silva LSM, Giugliani ERJ, Aerts DRGC. Prevalência e determinantes de anemia em crianças de Porto Alegre, RS, Brasil. Rev Saúde Pública. 2001; 35(1): 66-73. doi: 10.1590/S0034-89102001000100010.

16. Almeida CAN, Ricco RG, Del Ciampo LA, Souza AM, Pinho AP, Oliveira JED. Fatores associados a anemia por deficiência de ferro em crianças pré-escolares brasileiras. J Pediatr. 2004; 80(3): 229-34. doi: 10.1590/S0021-75572004000400012.

17. Bueno MB, Selem SSC, Áreas JAG, Fisberg RM. Prevalência e fatores associados à anemia entre crianças atendidas em creches públicas de São Paulo. Rev Bras Epidemiol. 2006; 9(4):462-70. doi: 10.1590/S1415-790X2006000400007.

18. Matta IEA, Veiga GV, Baião MR, Santos MMAS, Luiz RR. Anemia em crianças menores de cinco anos que frequentam creches públicas do município do Rio de Janeiro, Brasil. Rev Bras Saúde Mater Infant. 2005; 5(3):349-57. doi: 10.1590/\$1519-38 292005000300011.

19. Hadler MC, Juliano Y, Sigulem DM. Anemia do lactente: etiologia e prevalência. J Pediatr. 2002; 78(4):321-6. doi: 10.1590/S0021-755720020004 00012 .

20. Silva DG, Franceschini SC, Priore SE, Ribeiro SMR, Szarfarc SC, Souza SB, et al. Anemia ferropriva em crianças de 6 a 12 meses atendidas na rede pública de saúde do município de Viçosa, Minas Gerais. Rev Nutr. 2002; 15(3):301-8. doi: 10.1590/\$1415-52 732002000300006.

21. Silva DG, Priore SE, Franceschini SCC. Fatores de risco para anemia em lactentes atendidos nos serviços públicos de saúde: a importância das práticas alimentares e da suplementação com ferro. J Pediatr. 2007; 83(2):149-56. doi: 10.1590/\$0021-75 572007000200009.

22. Miranda AS, Franceschini SCC, Priore SE, Euclydes MP, Araújo RMA, Ribeiro SMR, et al. Anemia ferropriva e estado nutricional de crianças com idade de 12 a 60 meses do município de Viçosa, MG. Rev Nutr. 2003; 16(2):163-9. doi: 10.1590/S1415-5 2732003000200003.
23. Torres MAA, Lobo NF, Sato K, Queiroz SS. Fortificação do leite fluido na prevenção e tratamento da anemia carencial ferropriva em crianças menores de 4 anos. Rev Saúde Pública. 1996; 30(4):350-7. doi: 10.1590/S0034-89101996000400008.

24. Nogueira-de-Almeida CA, Ricco RG, Del Ciampo LA, Souza AM, Dutra-de-Oliveira JE. Growth and hematological studies on brazilian children of low socioeconomic level. ALAN. 2001; 51(3):230-5.

25. Vitolo MR, Bortolini GA, Feldens CA, Drachler ML. Impactos da implementação dos dez passos da alimentação saudável para crianças: ensaio de campo randomizado. Cad Saúde Pública. 2005; 21(5):1448-57. doi: 10.1590/S0102-311X20050 00500018.

26. Uchimura TT, Szarfarc SC, Latorre MRDO, Uchimura NS, Souza SB. Anemia e peso ao nascer. Rev Saúde Pública. 2003; 37(4):397-403. doi: 10.1590/S00 34-89102003000400002.

27. Neves MBP, Silva EMK, Morais MB. Prevalência e fatores associados à deficiência de ferro em lactentes atendidos em um centro de saúde-escola em Belém, Pará, Brasil. Cad Saúde Pública. 2005; 21(6): 1911-8. doi: 10.1590/S0102-311X2005000600041.

28. Leal LP, Osório MM. Validação e reprodutibilidade de sinais clínicos no diagnóstico de anemia em crianças. Cad Saúde Pública. 2005; 21(2):565-72. doi: 10.1590/S0102-311X2005000200023.

29. Spinelli MGN, Marchioni DML, Souza JMP, Souza SB, Szarfarc SC. Fatores de risco para anemia em crianças de 6 a 12 meses no Brasil. Rev Panam Salud Publica. 2005; 17(2):84-91. doi: 10.1590/S10 20-49892005000200004.

30. Szarfarc SC, Souza SB, Furumoto RAV, Brunken GS, Assis AMO, GaudenziEM, et al. Concentração de hemoglobina em crianças do nascimento até um ano de vida. Rev Saúde Pública. 2004; 20(1): 266-74. doi: 10.1590/S0102-311X20040001000 44.

31. Castro TG, Campos FM, Priore SE, Coelho FMG, Campos MTFS, Franceschini SCC, et al. Saúde e nutrição de crianças de 0 a 60 meses de um assentamento de reforma agrária, Vale do Rio Doce, MG, Brasil. Rev Nutr. 2004; 17(2):167-76. doi: 10.1590/S 1415-52732004000200003.

32. Morais MB, Alves GM, Fagundes-Neto U. Estado nutricional de crianças índias terenas: evolução do peso e estatura e prevalência atual de anemia. J Pediatr. 2005; 81(5):383-9. doi: 10.1590/S0021-7 5572005000600008 .

33. Orellana JDY, Coimbra Jr CEA, Lourenço AEP, Santos RV. Estado nutricional e anemia em crianças Suruí, Amazônia, Brasil. J Pediatr. 2006; 82(5): 383-8. doi: 10.1590/S0021-75572006000600013.

34. Ferreira HS, Assunção ML, Vasconcelos VS, Melo FP, Oliveira CG, Santos TO. Saúde de populações 
444 | R.C.S. VIEIRA \& H.S. FERREIRA

marginalizadas: desnutrição, anemia e enteroparasitoses em crianças de uma favela do "Movimento dos Sem Teto", Maceió, Alagoas. Rev Bras Saude Mater Infant. 2002; 2(2):177-85. doi: 10.15 90/S1519-38292002000200010.

35. Santos I, Cesar JA, Minten G, Valle N, Neumann $N A$, Cercato $E$. Prevalência e fatores associados à ocorrência de anemia entre menores de seis anos de idade em Pelotas, RS. Rev Bras Epidemiol. 2004; 7(4):403-15. doi: 10.1590/S1415-790X20040004 00004.

36. Vitolo MR, Bortolini GA. Biodisponibilidade do ferro como fator de proteção contra anemia entre crianças de 12 a 16 meses. J Pediatr. 2007; 83(1): 33-8. doi: 10.1590/S0021-75572007000100007.

37. Assis AMO, Santos LMP, Martins MC, Araújo MPN, Amorim DQ, Morris SS, et al. Distribuição da anemia em pré-escolares do semi-árido da Bahia. Cad Saúde Pública. 1997; 13(2):237-44. doi: 10.1590/S0102-3 11X1997000200013.

38. Assis AMO, Barreto ML, Gomes GSS, Prado MS, Santos NS, Santos LMP, et al. Childhood anemia prevalence and associated factors in Salvador, Bahia, Brazil. Cad Saúde Pública. 2004; 20(6):163 3-41. doi: 10.15 90/50102-311X20040006000 22

39. Osório, MM, Lira PIC, Batista-Filho M, AshWorth A. Prevalence of anemia in children 6-59 months old in the state of Pernambuco, Brazil. Rev Panam Salud Publica. 2001; 10(2):101-7. doi: 10.1590/S1 020-49892001000800005.

40. Muniz PT, Castro TG, Araújo TS, Nunes NB, SilvaNunes M, Hoffmann EHE, et al. Child health and nutrition in the Western Brazilian Amazon population-based surveys in two counties in Acre State. Cad Saúde Pública. 2007; 23(6):1283-93. doi: 10.1590/S0102-311X2007000600004.

41. Duarte LS, Fujimori E, Minagawa AT, Schoeps FA, Montero RMJM. Aleitamento materno e níveis de hemoglobina em crianças menores de 2 anos em município do estado de São Paulo, Brasil. Rev Nutr. 2007; 20(2): 149-57. doi: 10.1590/S1415-52732 007000200004 .

42. Neuman NA, Tanaka OY, Szarfarc SC, Guimarães PRV, Victora CG. Prevalência e fatores de risco para anemia no Sul do Brasil. Rev Saúde Saúde. 2000; 34(1):56-63. doi: 10.1590/\$0034-891020000001 00011.

43. Assunção MCF, Santos IS, Barros AID, Gigante DP, Victora CG. Anemia in children under six: population-based study in Pelotas, Southern Brazil. Rev Saúde Pública. 2007; 41(3):328-35. doi: 10.1590/S0034-89102007000300002.

44. DeMaeyer $E$, Adiels-Tegman M. The prevalence of anaemia in the world. World Health Stat Q. 1985; 38(3):302-16.
45. Simmons WK. Control de la deficiencia de hierro en el Caribe de habla inglesa/Control of iron deficiency in the English-speaking Caribbean. Bol Oficina Sanit Panam.1994; 117(6):538-46.

46. Batista-Filho M, Souza Al, Bresani CC. Anemia como problema de saúde pública - uma realidade atual. Cien Saude Colet. 2007; 13(6):1917-22. doi: 10.1590/S1413-81232008000600027.

47. Batista Filho $M$, Rissin A. A transição nutricional no Brasil: tendências regionais e temporais. Cad Saúde Pública. 2003; 19(Supl 1):181-91. doi: 10.15 90/S0102-311X2003000700019.

48. Vieira RCS, Ferreira HS, Costa ACS, Moura FA, Florêncio TMMT, Torres ZMC. Prevalência e fatores de risco para anemia em crianças pré-escolares do Estado de Alagoas, Brasil. Bras Saúde Mater Infant. 2010; 10(1):107-16. doi: 10.1590/S1519-382920 10000100011.

49. Lima ACVMS, Lima MC, Guerra MQF, Romani; Eickmann SH, Lira PIC. Impacto do tratamento semanal com sulfato ferroso sobre o nível de hemoglobina, morbidade e estado nutricional de lactentes anêmicos. J Pediatr. 2006; 82(6):452-7. doi: 10.1590/S0021-75572006000800010.

50. Agência Nacional de Vigilância Sanitária. Resolução n. 344. Revoga a resolução - RDC no 15, de 21 de fevereiro de 2000. Diário Oficial da União, 2002.

51. Ghosh K. Non haematological effects of iron deficiency: a perspective. Indian J Med Sci. 2006; 60(1):30-7.

52. Oliveira RS, Diniz AS, Benigna MJC, Miranda-Silva SM, Lola MM, Gonçalves MC, et al. Magnitude, distribuição espacial e tendência da anemia em pré-escolares da Paraíba. Rev Saúde Pública. 2002; 36(1):26-32. doi: 10.1590/S0034-891020020001 00005.

53. Escoda MSQ. A determinação social da fome e a intervenção do Estado [dissertação]. Natal: Universidade Federal do Rio Grande do Norte; 1989.

54. Oliveira MAA, Osório MM. Consumo de leite de vaca e anemia ferropriva na infância. J Pediatr. 2005; 81(5):361-7. doi: 10.1590/S0021-75572005000 600004.

55. Conselho Nacional de Segurança Alimentar e Nutricional. Conferência nacional de segurança alimentar e nutricional: relatório final. Fortaleza: CONSEA; 2007. Disponível em: <https://www. planalto.gov.br/consea/3conferencia/static/ Documentos/RelatorioFinal.pdf>.

Recebido em: 14/4/2008

Versão final reapresentada em: 24/2/2010 Aprovado em: 29/4/2010 